\title{
Dysregulation of IL-4 expression in lepromatous leprosy patients with and without erythema nodosum leprosum
}

\author{
I. NATH*, N. VEMURI*, A. L. REDDI*, \\ M. BHARADWAJ*, P. BROOKS $\dagger$, M. J. COLSTON $\dagger$, \\ R. S. MISRA** \& V. RAMESH** \\ *Department of Biotechnology, All India Institute of Medical \\ Sciences, **Department of Dermatology, Safdarjung Hospital, New \\ Delhi, India, and †National Institute for Medical Research, Mill Hill, \\ London, UK
}

\begin{abstract}
Summary In order to increase our understanding of the immunological basis of erythema nodosum leprosum (ENL), we studied Th-like cytokine profiles in 130 leprosy patients, employing both the conventional and a novel, real-time, fluorogenic reverse transcriptase-based PCR (RT-PCR). The concomitant expression of both Thlike cytokines, interferon- $\gamma$ and IL-4, and the regulatory cytokines, IL-10 and IL-12, was studied in the peripheral blood cells of leprosy patients with and without ENL. In the conventional RT-PCR, varied cytokine profiles were observed in individual patients of all clinical types. Fifty-three percent of lepromatous patients without ENL and $59 \%$ of tuberculoid leprosy patients showed co-expression of IFN $\gamma$ and IL-4, indicating a non-polarized Th 0 pattern. Of the 36 patients with ENL, 58\% demonstrated a polarized Th 1 pattern, with only $30 \%$ expressing both cytokines. Semiquantitative RT-PCR indicated a lower expression of IL-4 compared to that of IFN $\gamma$ in the lepromatous patients without ENL; the difference was even greater among those with ENL. The sensitive, real-time PCR confirmed the down-regulation of IL-4 and IL-10, with absence of IL-4 in half of the patients, resulting in skewing of the cytokine response toward a Th 1-like profile.
\end{abstract}

\section{Introduction}

The mechanisms underlying the anergy associated with lepromatous leprosy continue to elude consensus in the scientific community. The discovery of subsets of T cells, which are definable on the basis of their cytokine profiles, has presented the possibility of understanding the leprosy spectrum and the antigen-specific T-cell-related unresponsiveness. Both CD4+ and CD8+ T cells may be divided into three populations. Th 1 cells produce interleukin-2 (IL-2) and interferon- $\gamma$ (IFN $\gamma$ ), and appear to be important for the development of cellmediated immunity and resistance to intracellular pathogens. Th 2 cells produce IL-4, IL-5, IL-6 and IL-13, and regulate antibody production and immunoglobulin subclass differentiation, thereby playing a role in protection against extracellular pathogens. Th 0 cells, the function of which is less clear, produce both sets of cytokines. ${ }^{1}$ Although these T-cell subsets 
are clearly demonstrable in the mouse, a polarized Th 2 profile has been seen in man only in helminthic infestations and allergic states. ${ }^{2}$

The leprosy spectrum represents an ideal model for study of the role of these subsets, as it shows an inverse relationship between cell-mediated and humoral immune responses, suggesting a Th 1 and Th 2 dichotomy. However, reports of earlier work on leprosy patients, including ours, have shown a varied Th-like cytokine pattern in both peripheral blood mononuclear cells (PBMC) and lesions, ranging from polarized Th 1 and Th 2 patterns to a mixed pattern, in which both Th 1 and Th 2 subsets or all three subsets have been observed. $^{3-5}$ On the other hand, consensus exists with regard to the reactional states in leprosy, in which most patients with both reversal (type 1) and erythema nodosum leprosum (ENL) (type 2) reactions have been shown to have a polarized Th 1-like cytokine pattern. ${ }^{6-9}$ It is not clear whether the patients without ENL with Th 0 and Th 2 patterns undergo phenotypic change to Th 1 during ENL. It is also not clear whether the diversity of findings in different laboratories has resulted from the use of less sensitive conventional RT-PCR methods to identify cytokine mRNA, and the use of supernatants from the mixed population of PBMC to measure the cytokine product by ELISA.

The present study was undertaken in an attempt to increase our understanding of the change of Th-profile that occurs in lepromatous patients undergoing ENL, employing a comparison of the conventional with the more recently developed fluorogenic, real-time RT-PCR ${ }^{10,11}$ to measure the relative expression of the cytokines that define the Th-subsets.

\section{Materials and methods}

\section{SUBJECTS}

One hundred and thirty leprosy patients (69 LL/BL, $18 \mathrm{BT}$, seven with reversal reaction, and 36 with ENL), who attended the clinics of Safdarjung and Leprosy Mission Hospitals in New Delhi, were included in the study. The 18 patients with tuberculoid leprosy, together with nine healthy contacts with history of exposure to leprosy patients, were included for comparison of anergic and responsive clinical types. Lepromatous and tuberculoid patients were under treatment for from 4 to 24 weeks by the standard multidrug regimen of $100 \mathrm{mg}$ dapsone daily, $100 \mathrm{mg}$ clofazamine on alternate days, and $600 \mathrm{mg}$ of rifampicin monthly. The patients with ENL were tested prior to anti-reaction therapy.

PBMC CULTURES FOR CYTOKINE MRNA

PBMC were isolated from the subjects as before, ${ }^{12}$ and cultured for $24 \mathrm{~h}$ at $4 \times 10^{6}$ cells per ml per well in 24-well plates in RPMI-1640 cell-culture medium containing $10 \%$ human AB serum, with or without $10^{7}$ integral Mycobacterium leprae. Cytokine mRNA was isolated from the cultures of PBMC, reverse-transcribed to cDNA, and stored at $-20^{\circ} \mathrm{C}$ as described earlier. ${ }^{3}$

CONVENTIONAL RT-PCR

cDNA was amplified for 35 cycles, except where otherwise indicated, using cytokine-specific primers, cycling parameters and ethidium bromide-stained agarose gels for visualization of amplicons, as described earlier. ${ }^{3}$ Semiquantitative PCR involved amplification of each cDNA for $20,25,30$ and 35 cycles, employing the same quantity of cDNA and the same primers. 
FLUOROGENIC REAL-TIME PCR

The cDNA samples were transported on dry ice to the UK laboratory. Cytokine- and $\beta$-actinspecific primers, which were designed to avoid genomic DNA contamination with the aid of Gene Express software, were obtained from PE Applied Biosystems, UK. Plasmids containing genes encoding $\beta$-actin, IFN $\gamma$, IL-4, IL-10, and IL-20p40, constructed in pGEM-T vector (Promega, Madison, Wisconsin, USA), and a known patient cDNA sample served as positive controls. PCR amplification was carried out in a volume of $25 \mu 1$, using a Taqman PCR kit according to the manufacturer's instructions, and the fluorescence spectra were detected in the ABI Prism 7700 sequence detection system (Perkin-Elmer Applied Biosystems). One $\mu \mathrm{l}$ of cDNA reverse-transcribed from $1 \mathrm{~g}$ total RNA was added to triplicate tubes, together with $20 \mu$ l of a master mixture of previously determined optimal quantities of forward and reverse primers and an FAM-labelled cytokine-probe. The amplification parameters consisted of a hold-cycle of $2 \mathrm{~min}$ at $50^{\circ} \mathrm{C}$ to activate the uracil $\mathrm{N}^{\prime}$-glycosylase, followed by $10 \mathrm{~min}$ at $95^{\circ} \mathrm{C}$ and 40 cycles of $95^{\circ} \mathrm{C}$ for $15 \mathrm{~s}$ and $60^{\circ} \mathrm{C}$ for $1 \mathrm{~min}$. Real-time fluorescence measurements were taken every $8 \mathrm{~s}$, and a value for the threshold cycle $\left(\mathrm{C}_{\mathrm{T}}\right)$ of each sample was calculated by determining the cycle at which normalized fluorescence exceeded the threshold limit of 10 times the SD of the baseline, using Perkin-Elmer Applied Biosystems Version 1.6 software. The relative expression of the various cytokines was compared continuously during the logarithmic phase of PCR, in terms of $\Delta C_{T}$ (mean $C_{T}$ of three replicate amplifications of cytokine cDNA - mean $\mathrm{C}_{\mathrm{T}}$ of three replicate amplifications of $\beta$-actin cDNA). Samples were considered negative when the signal did not attain threshold levels within 40 cycles. $\beta$-Actin levels were measured in each sample using commercial standards of human DNA (Perkin-Elmer Applied Biosystems).

\section{Results}

The Th-like phenotypes detected by the conventional RT-PCR up to 35 amplification cycles are shown in Table 1. It may be observed that approximately half of the lepromatous patients demonstrated variation of the proportion of each Th-subset. Approximately half of the lepromatous patients without ENL and the tuberculoid patients showed the presence of the non-polarized Th 0-phenotype in the antigen-stimulated PBMC, indicating the concomitant presence of both IFN $\gamma$ and IL-4 mRNA. Whereas $31 \%$ of the tuberculoid patients demonstrated a Th 1 profile, a similar proportion of the lepromatous patients demonstrated a Th 2 profile. Small proportions of patients showed the opposite Th-subset or absence of both cytokines. Importantly, the majority of lepromatous patients with reversal and ENL reactions

Table 1. Th phenotypes in PBMC of leprosy patients by RT-PCR

\begin{tabular}{lcccc}
\hline & & & & \\
Patients & Th 0 & Th 1 & Th 2 & Th-negative \\
\hline Lepromatous (69) & 54 & 7 & 33 & 4 \\
Tuberculoid (18) & 59 & 31 & 10 & 0 \\
Reversal reactions (7) & 14 & 86 & 0 & 0 \\
ENL (36) & 30 & 58 & 0 & 11 \\
& & & & \\
\hline
\end{tabular}




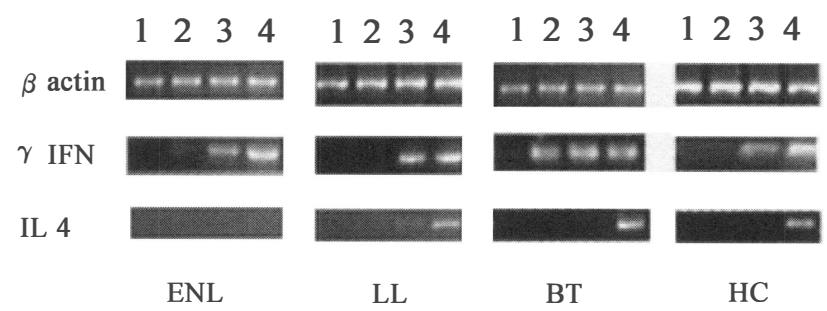

Figure 1. Conventional PCR. Amplification of $\beta$-actin, interferon- $\gamma$ (IFN $\gamma)$ and IL-4 in the same cDNA sample obtained from antigen-stimulated peripheral blood mononuclear cells of leprosy patients. PCR products were amplified for: (1) 20; (2) 25; (3) 30; and (4) 35 cycles, and were visualized in ethidium bromide-stained gels under UV light. Note the earlier amplification of IFN $\gamma$ than of IL 4. Abbreviations: LL= polar lepromatous leprosy; $\mathrm{ENL}=$ erythema nodosum leprosum; $\mathrm{BT}=$ borderline tuberculoid; $\mathrm{HC}=$ household contacts.

showed a Th 1-like pattern, with the presence of IFN $\gamma$ mRNA and the absence of IL-4 mRNA. Fewer subjects demonstrated Th- 0 subsets.

To determine the relative numbers of copies of the mRNA of each cytokine, we undertook a semiquantitative RT-PCR, whereby the same quantity of cDNA of each patient was amplified at fixed cycle times of $20,25,30$ and 35, with the expectation that larger numbers of copies of the mRNA would amplify at an earlier cycle time. Figure 1 is representative of the cytokine signals obtained for one patient from each clinical type in ethidium bromide-stained agarose gels. The $\beta$-actin mRNA was visualised earliest, between 20 and 25 cycles. By 35 cycles, all clinical types showed a Th 0-like cytokine profile, with concomitant signals for both Th 1 and Th 2 cytokines. However, differences became apparent when earlier cycle times were tested. IFN $\gamma$ mRNA was detected earlier in tuberculoid leprosy than in other clinical types. Furthermore, there were relatively more IFN $\gamma$ mRNA copies, with signals detected at 25 cycles, compared to 30 cycles for IL-4 mRNA. The healthy contact showed a similar pattern, but demonstrated a smaller number of IFN $\gamma$ mRNA copies than did the tuberculoid patient. The LL patient showed both cytokines by 30 cycles, indicating a Th 0 profile; it was not possible to determine the relative numbers of copies of the two cytokines.

By contrast, the patient with ENL showed a larger copy number of IFN $\gamma$ mRNA (20 cycles), compared to all other clinical types. Moreover, IL-4 was not detectable even at 35 cycles, indicating smaller copy numbers and a down-regulation of expression, compared to the patient without ENL. Therefore, it is apparent that quantitative differences of the Th 1 and Th 2 cytokines are present among patients with different clinical forms of leprosy; ENL patients show a further lowering of IL-4, with skewing of the response toward the Th 1-type, compared to the lepromatous patient without ENL.

To quantify the relative proportions of Th1 and 2 cytokine expression more accurately, we used the recently developed real-time PCR, which uses a fluorogenic probe for detection of amplified product at each cycle time. Figure $2 \mathrm{a}$ shows a representation of the relative expression of IFN $\gamma$ and IL-4 in a patient with ENL. The amplification dynamics were similar for both types of cDNA, indicating that the observed differences chiefly reflected differences of mRNA copy-numbers in the PBMC. The copy-number of $\beta$-actin mRNA was larger than that of cytokine mRNA, and reached threshold after 20 cycles of amplification. In agreement with the data from agarose gels, IFN $\gamma$ reached threshold level after 26 cycles, whereas IL-4 was detectable only after 36 cycles. Among the regulatory cytokines (see Figure 2b), IL-12 was detected earlier ( 25 cycles) than was IL-10 (30 cycles). In Table 2, the data are presented 

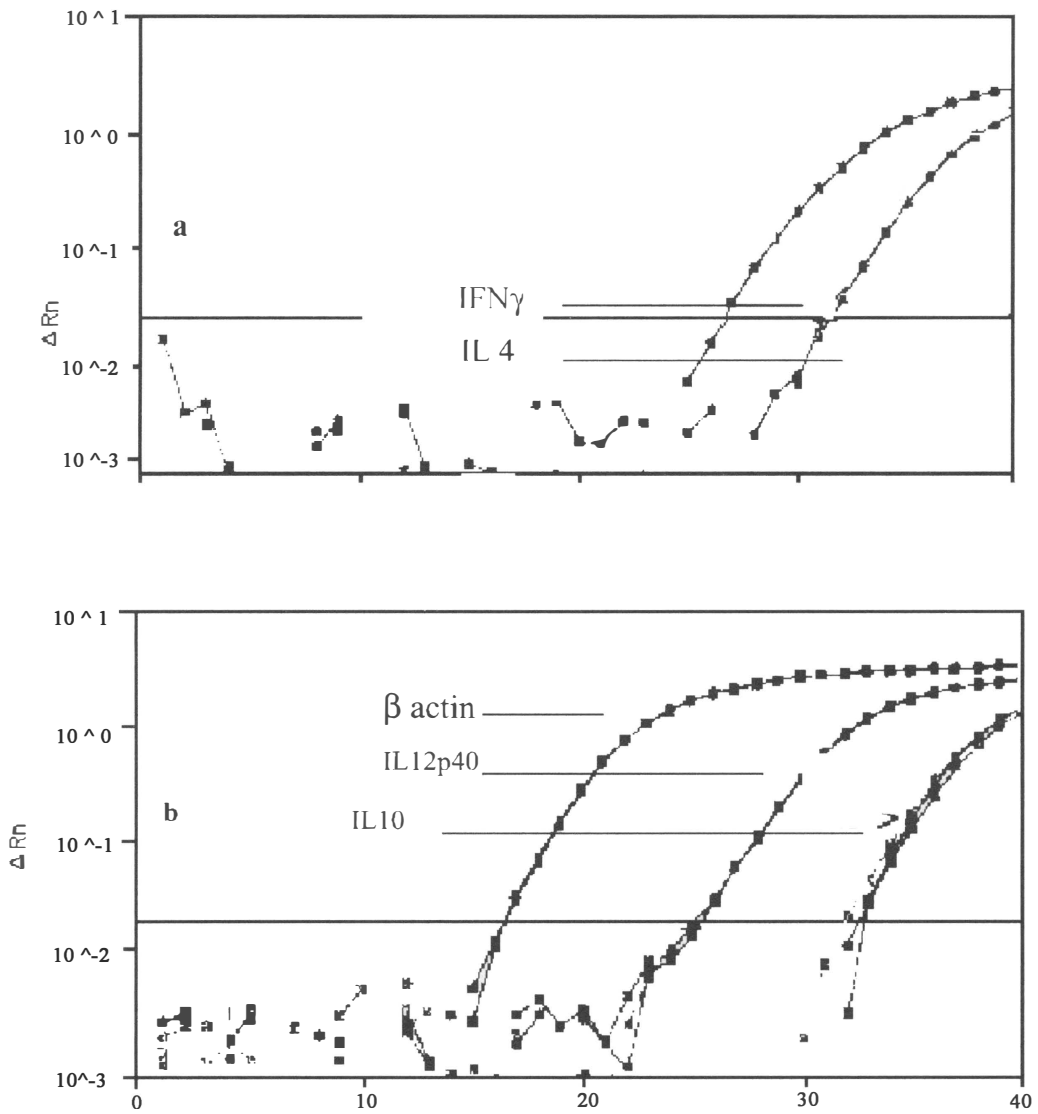

Figure 2. Real-time PCR. Amplification plots of (a) interferon- $\gamma$ (IFN $\gamma$ ) and IL-4; (b) $\beta$-actin, IL-10 and IL-12p40 from antigen-stimulated PBMC of an LL patient without ENL. In real-time PCR, the cytokines are shown with their respective $\log$ phases indicated by lines. The $\mathrm{X}$-axis indicates the cycle time $\left(\mathrm{C}_{\mathrm{T}}\right)$ of amplification. The $\mathrm{Y}$-axis shows normalized fluorescence $(\Delta \mathrm{Rn})$ of the reporter dye. The horizontal line indicates the threshold $\Delta \mathrm{Rn}$, and is $10 \mathrm{SD}$ above the baseline. Abbreviations: $\mathrm{LL}=$ polar lepromatous leprosy; $\mathrm{ENL}=$ erythema nodosum leprosum; $\mathrm{PBMC}=$ peripheral blood mononuclear cells; $\mathrm{IL}=$ interleukin.

as mean $2^{-\Delta \mathrm{CT}}$ for each group of leprosy patients. Among the lepromatous patients, the relative decrease of IL- 4 compared to IFN $\gamma$ was evident when both cytokines were expressed. In agreement with these findings, the number of copies of IL-10 mRNA was smaller than that of IL-12p40. These cytokines are known to help, respectively, in Th 2- and Th 1-subset differentiation after antigen-exposure or infection. ${ }^{1}$

Confirming the earlier findings by conventional RT-PCR, ENL patients showed not only spontaneous expression of IFN $\gamma$ in unstimulated ex-vivo cells, but also a further downregulation of IL-4-expression, which rendered its mRNA undetectable, even using this sensitive assay. Thus, the Th1 polarization pattern, which is associated with the immunopathology in patients with ENL, appears to result from dysregulation or down-regulation of IL-4. Furthermore, expression of IFN $\gamma$ was highly variable in patients with active ENL, indicating an immune response in a dynamic state. 
Table 2. Relative numbers of copies $\left(2^{-\Delta C T}\right)$ of cytokine genes in PBMC of lepromatous patients with and without ENL

\begin{tabular}{|c|c|c|c|c|}
\hline \multirow[b]{2}{*}{ Patients } & $\mathrm{IFN} \gamma$ & IL-4 & IL-10 & IL-12p40 \\
\hline & \multicolumn{4}{|c|}{ Mean \pm SD (range) } \\
\hline $\begin{array}{l}\text { BL/LL } \\
\text { (13) }\end{array}$ & $\begin{array}{l}0.334 \pm 0.2 \\
(0-0.65)\end{array}$ & $\begin{array}{l}0.002 \pm 0.003 \\
(0-0.01)\end{array}$ & $\begin{array}{l}0 \cdot 103 \pm 0 \cdot 135 \\
(0-0 \cdot 4)\end{array}$ & $\begin{array}{l}0.901 \pm 2.67 \\
(0.02-9.8)\end{array}$ \\
\hline $\begin{array}{l}\text { ENL } \\
(8)\end{array}$ & $\begin{array}{l}30 \cdot 8 \pm 52 \cdot 8 \\
(0 \cdot 001-128)\end{array}$ & $\begin{array}{l}0 \cdot 102 \pm 0 \cdot 266 \\
(0-0 \cdot 76)\end{array}$ & $\begin{array}{l}0.252 \pm 0.367 \\
(0-0.95)\end{array}$ & $\begin{array}{l}166 \pm 264 \\
(0-630)\end{array}$ \\
\hline
\end{tabular}

The values in brackets indicate the ranges.

\section{Discussion}

In order to understand the relationship of Th phenotypes to the clinical types of leprosy, as well as the emergence of reactional states in hitherto anergic subjects, we used RT-PCR to amplify the cytokine mRNA. In view of the various reports on Th-phenotypes in leprosy patients, we used both the conventional and the recently developed, highly sensitive, realtime RT-PCR to quantify cytokine mRNA. In general, the cytokine profiles obtained by both methods were comparable; the new method possessed the added advantage of a bettercontrolled and more sensitive assay, which was amenable to simultaneous quantification of multiple cytokine mRNA molecules in several patient samples.

In conventional RT-PCR, the predominant profile in all clinical groups was that of Th 0 . Approximately one-third of lepromatous patients expressed IL-4 exclusively. By examining earlier cycle-times in the semiquantitative RT-PCR, and exploiting the increased sensitivity of the real-time PCR, both cytokines were detectable in all lepromatous patients without ENL. Of interest was the dominance of IFN $\gamma$, which showed several-fold greater expression than IL-4, when both cytokines were expressed. IFN $\gamma$ was detectable as early as 26-28 cycles, and reached a plateau in all samples by 30 cycles. By contrast, IL- 4 was detectable after 30-38 cycles. The low expression of IL-4 could not be attributed to time-kinetics or the efficiency of PCR, as plasmid DNA and control cDNA reached threshold levels between 27 to 28 and 32 to 33 cycles, respectively, in most experiments. The differences of IFN $\gamma$ and IL-4 levels in the present study were not attributable to time-kinetics, as they were observed in antigen-stimulated cultures incubated for 12 or $48 \mathrm{~h}$.

The relative decrease of IL- 4 and the dominance of IFN $\gamma$ were unexpected; the biology of the disease suggests a polarized Th 2-like response, because lepromatous patients demonstrate high antibody levels and T-cell anergy to the antigens of $M$. leprae. ${ }^{13}$ However, even by this sensitive method, IL-4 was not detected in patients during the immunological perturbations associated with ENL, thus skewing the Th-profile to a polarized Th 1phenotype, consistent with previous reports on ENL reactions from our and other laboratories. ${ }^{4,-9}$ Moreover, when both cytokines were expressed in some subjects, IL-4 mRNA copy numbers were much smaller than those of IFN $\gamma$ mRNA. Antigen-stimulated cultures showed greater variability of IFN $\gamma$ expression-levels among patients with ENL, indicating a dynamic disease state, in which individuals are at different levels of an ongoing immune response. Spontaneous expression of IFN $\gamma$ but not of IL-4 was observed in unstimulated PBMC, consistent with in-vivo T-cell priming in ENL.

Consistent with the development of a polarized Th 1-response, emergence of transient, 
antigen-specific T-cell reactivity has been observed in ENL. ${ }^{14}$ Paradoxically, ENL patients also have high levels of anti- $M$. leprae antibody and immune complexes. ${ }^{13,15}$ Although Thsubsets influence Ig isotypes and subclasses in experimental models ${ }^{1}$ and allergic states, ${ }^{2}$ their role in human infections is less clear. ENL appears to be associated with low levels of IgG-3 antibodies to several antigens of $M$. leprae antigens. ${ }^{16}$

IL-12 and IL-10 regulate the immune response by influencing the induction, respectively, of type 1 and type 2 cytokines during infection. ${ }^{1}$ In agreement with the relatively greater decrease of IL-4 than of IFN $\gamma$, expression of IL-10 was decreased in patients with ENL to a significantly greater degree than was expression of IL-12p40. Predominance of IL-12p40 during reaction could result in an increase of IFN $\gamma$ and down-regulation of IL-4, thereby skewing the immune response to the Th 1 type. In conclusion, human diseases such as lepromatous leprosy without ENL appear not to show true Th 1- or Th 2-polarization in the peripheral blood. Nevertheless, perturbations of Th 1- or Th 2-like cytokines may lead to the transient clinical changes observed in leprosy reaction. The factors triggering such cytokine alterations should be defined, if better strategies for control of ENL are to be developed. The administration of recombinant IFN $\gamma$ leads to precipitation of ENL in some subjects, ${ }^{17}$ demonstrating the importance of cytokines in the clinical changes that occur during chronic infection.

\section{Acknowledgements}

This study was supported by the Department of Science and Technology (DST), Government of India, and the British Leprosy Relief Association (LEPRA). N. Vemuri and A. L. Reddi are Research Associates of the DST and the Indian National Science Academy. Real-time PCR studies in London were supported by a grant to I. Nath from the Cochrane Fund. The excellent technical help of Mr Manickam is gratefully acknowledged.

\section{References}

I O'Garra A, Murphy K. Role of cytokines in determining T-cell lymphocytic functions. Curr Opin Immunol, 1994; 6: $458-466$.

2 Romagnani S. Lymphokine production by human T cells in disease states. Annu Rev Immunol, 1994; 12: 227257.

${ }^{3}$ Misra N, Murtaza A, Walker B, Narayan NPS et al. Cytokine profile of circulating T cells of leprosy patients reflects both indiscriminate and polarized T-helper subsets: T-helper phenotype is stable and uninfluenced by related antigens of Mycobacterium leprae. Immunology, 1995; 86: 97-103.

4 Yamamura M, Uyemura K, Deans RJ et al. Defining protective responses to pathogens: cytokine profiles in leprosy lesions. Science, 1991; 254: 277-279.

5 Mutis T, Kraakman EM, Cornelisse Y et al. Analysis of cytokine production by Mycobacterium-reactive T cells. Failure to explain Mycobacterium leprae-specific nonresponsiveness of peripheral blood T cells from lepromatous leprosy patients. J Immunol, 1993; 150: 4641-4651.

${ }^{6}$ Verhagen CE, Wierenga EA, Buffing AAM et al. Reversal reaction in borderline leprosy is associated with a polarised shift to Type 1-like Mycobacterium leprae $\mathrm{T}$ cell reactivity in lesional skin: a follow-up study. $J$ Immunol, 1997; 159: 4474-4483.

7 Sreenivasan P, Misra RS, Wilfred D, Nath I. Lepromatous leprosy patients show T helper 1-like cytokine profile with differential expression of interleukin-10 during type 1 and 2 reactions. Immunology, 1998; 95: 529-536.

8 Sampaio EP, Samo EN. Expression and cytokine secretion in the states of immune reactivation in leprosy. Braz $J$ Med Biol Res, 1998; 31: 69-76.

9 Modlin RL. Th1-Th2 paradigm: insights from leprosy. J Invest Dermatol, 1994; 102: 828-832.

${ }^{10}$ Heid CA, Stevens J, Livak KJ, Williams PM. Real time quantitative PCR. Genome Res, 1996; 6: 986-994. 
11 Gibson UEM, Heid CA, Williams PM. A novel method for real time quantitative PCR. Genome Res, 1996; 6: 995-1001.

12 Misra N, Selvakumar M, Singh S et al. Monocyte derived IL10 and PGE 2 are associated with the absence of Th1 cells and in vitro T cell suppression in lepromatous leprosy. Immunol Lett, 1995; 48: 123-128.

13 Hastings RC (ed). Leprosy, 2nd edn. Churchill Livingstone, Edinburgh, 1994.

14 Laal S, Bhutani LK, Nath I. Natural emergence of antigen reactive T-cells in lepromatous leprosy patients during erythema nodosum leprosum. Infect Immun, 1985; 50: 887-892.

15 Singh S, Shanker Narayanan NP et al. Sera of leprosy patients with type 2 reactions recognize selective sequences in Mycobacterium leprae recombinant LSR protein. Infect Immun, 1994; 62: 86-90.

16 Hussain R, Menz BM, Dockrell HM, Chiang TJ. Recognition of Mycobacterium leprae recombinant 18000 MW epitopes by subclasses in leprosy. Immunology, 1995; 84: 290-297.

17 Sampaio EP, Moreira AL, Samo EN et al. Prolonged treatment with recombinant interferon gamma induces erythema nodosum leprosum in lepromatous leprosy patients. J Exp Med, 1992; 175: 1729-1737.

\section{DISCUSSION}

Dr Kaplan: Don't your results contradict some of those in the literature? I remember some earlier reports that suggested that the cytokine profile was different from that you have described, especially in lepromatous patients and those with ENL. Is this a function of the sensitivity of your assay, or are you dealing with different kinds of patients?

Professor Nath: I think my data from patients with ENL do not contradict those in the literature. There may well be a contradiction with respect to the results from patients with BL/ LL disease without ENL. I think that our assay is more sensitive so that it detects more of the other cytokines, so that patients who might have appeared Th 1 are actually Th 0 . I'm uncertain whether we're dealing with a mixture of Th 1 and Th 2 subsets or with a single cell that produces both types of cytokine. I'm inclined to believe that we're dealing with a mixture.

Dr Modlin: Our data showed that there was a mixture of cytokines in both forms of leprosy, with a predominance of Th 2 cytokines in tuberculoid lesions and a predominance of Th 1 cytokines in lepromatous lesions. I believe your data to be consistent with what has been published. In terms of comparing IFN $\gamma$ and IL- 4 mRNA, I should point out that it is easy to compare quantities of the same cytokine mRNA in two lesions, but one must be cautious in comparing quantities of different cytokines.

Professor Britton: You've shown us your results in BL and LL patients. Have you applied the same techniques to TT/BT patients?

Professor Nath: I haven't applied real-time PCR to tuberculoid patients. 